\title{
Early Prognostic Factors for Walking in Spinal Cord Injuries
}

\author{
P. Daverat, M. C. Sibrac, J. F. Dartigues, J. M. Mazaux, E. Marit, \\ X. Debelleix, M. Barat \\ Service de Re-education Fonctionnelle Neurologique, Centre Hospitalo-Univer- \\ sitaire-33076 Bordeaux Cedex, France.
}

\section{Summary}

The authors present a prospective analysis with 1 year follow-up of about 157 consecutive spinal cord injured patients admitted to the Bordeaux CHU, over a 3 year period (1982-1985). They present a method of analysing the walking status recovery in patients with spinal cord injuries from the early factors recorded on initial clinical examination. Twenty eight per cent of all patients are 'functional walkers', 1 year after injury. Three early factors were selected: age; the level of injury; and the initial Yale Scale Score. Only the age and the initial Yale Scale Score are independant predictive factors, according to the Cox model.

The authors present a model of walking status recovery, 1 year after the injury, predicted from age and the initial Yale Scale Score.

Key words: Spinal cord injury; Walking status; Prognosis.

The prediction of the evolution of a spinal cord injury is necessary for the physician to develop a therapeutic strategy for the patient for functional activity and for any economic repercussions. When the initial emergency is resolved, the question is to know to what level a spinal injured patient will recover a functional autonomy in his daily life. We chose as functional criteria the walking status recovery, for this is the most commonly studied in the literature. We have to acknowledge that the physician does not give a prognosis of the possibilities of late recovery, according to the severity of the initial spinal shock. Our team operates very early in the evaluation of these injured patients and collaborates with the emergency team. As we are involved in the earliest care of the patients, we are confronted with the question of the prognosis from the very day of the injury.

The purpose of this work is to establish a prediction for walking recovery in those with spinal cord injuries, from the early factors recorded on the initial clinical examination.

\section{Patients and methods}

The crucial point is to obtain a population as representative as possible of the 'theoretical' population of the spinal injured patients. If some selection bias exist, due to a defective protocol or simply to chance, the results may be totally wrong. To try to overcome the risk we consider the prognosis very early on on 
the day of the admission, in consecutive cases (Dartigues, 1985). Only patients who die on the street or during transfer to hospital will escape from our analysis. It represents a minimal selection. Our results will not be comparable to those of the specialised departments of re-education or re-adaptation centres. Their samples are much more selected, which increases the positive results.

We included in the study 157 patients, consecutively admitted between January 1982 and July 1985 to the emergency room of the Pellegrin Hospital in Bordeaux, who had sustained a spinal injury. We studied the walking status recovery in a prospective analysis over a minimal follow-up period of 1 year. Six of the 157 patients were lost to follow-up within the first month, 5 during the following 6 months.

In the present study, walking status is defined as adequate motor power in the legs allowing patients to be functional walkers with or without orthoses. A functional walk will be either almost normal or limited to walking inside the house.

The following step is the selection of the early factors, which may play a role in the walking recovery. According to the literature, we selected three factors. Such a choice may appear restrictive but increasing the number of the factors would decrease the value of the statistical analysis.

We selected three factors: age, the level of the injury and the initial Yale Scale Score (YSS) (Chehrazi et al., 1981). The YSS is a quantitative measurement which does not have the ambiguous characters of the qualitative scores generally used (i.e. Frankel Classification). The YSS is determined by motor and sensory testing, each on a scale of five points. Each patient will thus have a score from 0 to 10 . Zero indicates a complete lesion without any motor and sensory function below the level of the injury. Ten stands for normal spinal neurological function.

The interest of the YSS is its simplicity and its reliability. It is easy to apply in the often dramatic conditions of the emergency room.

The last methodological step is the statistical analysis, which will be discussed later in this paper.

\section{Results}

\section{Description of the population}

Figure 1 gives the age distribution of the patients. The mean age is 38 years. Forty one per cent of the patients are in the range of 20 to 40 . However, $42^{\circ}{ }_{0}$ of the population are over 40 ; thus a spinal injury is not confined to young people.

The distribution of the patients according to the level of the injury is shown in Figure 2. Cauda equina syndromes are excluded from this study.

Fifty two per cent of the population were tetraplegic. Only $3^{\circ}{ }_{0}$ of the lesions were at the $\mathrm{C} 1-\mathrm{C} 2-\mathrm{C} 3$ level, because most of them died before reaching hospital. Forty per cent were at the level of C4-C5-C6. Among the paraplegic patients $\left(48^{\circ}{ }_{0}\right.$ of the population) the lesions are mainly found at T3-T4 $\left(11^{\circ}{ }_{0}\right)$ and $\mathrm{T} 11-$ T12 (13\%).

Fifty per cent of the patients have a complete lesion (YSS $=0$ ). Forty seven per cent among the tetraplegics and $53^{\circ}$ o among the paraplegics are complete. The almost equal number of paraplegics and tetraplegics with complete lesion 


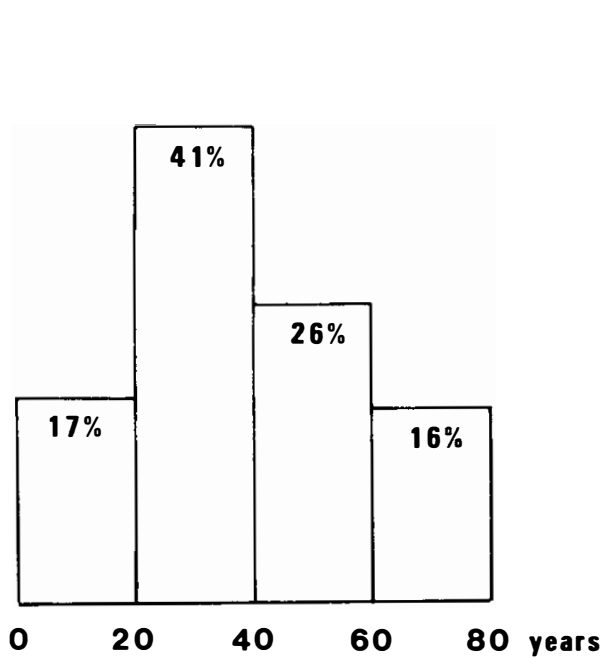

Figure 1 Age distribution of the 157 patients.

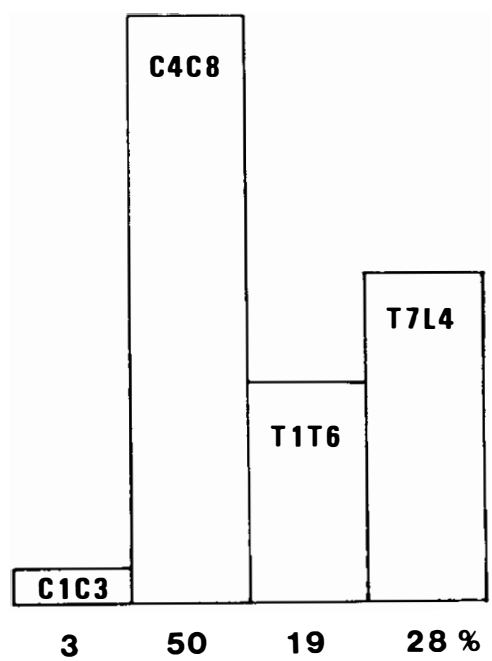

Figure 2 Distribution of the 157 patients according to the level of the injury.
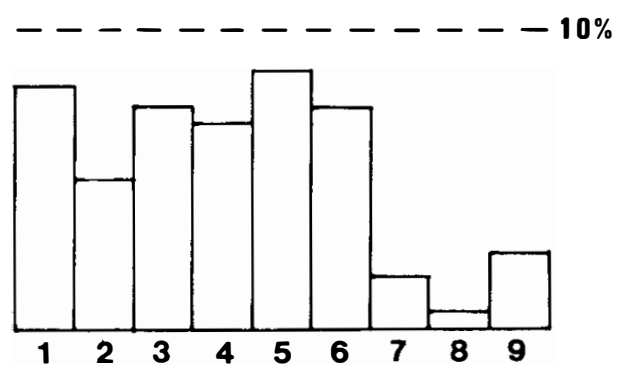

Figure 3 Distribution of the YSS among the incomplete lesions $(1<$ YSS $<9)$.

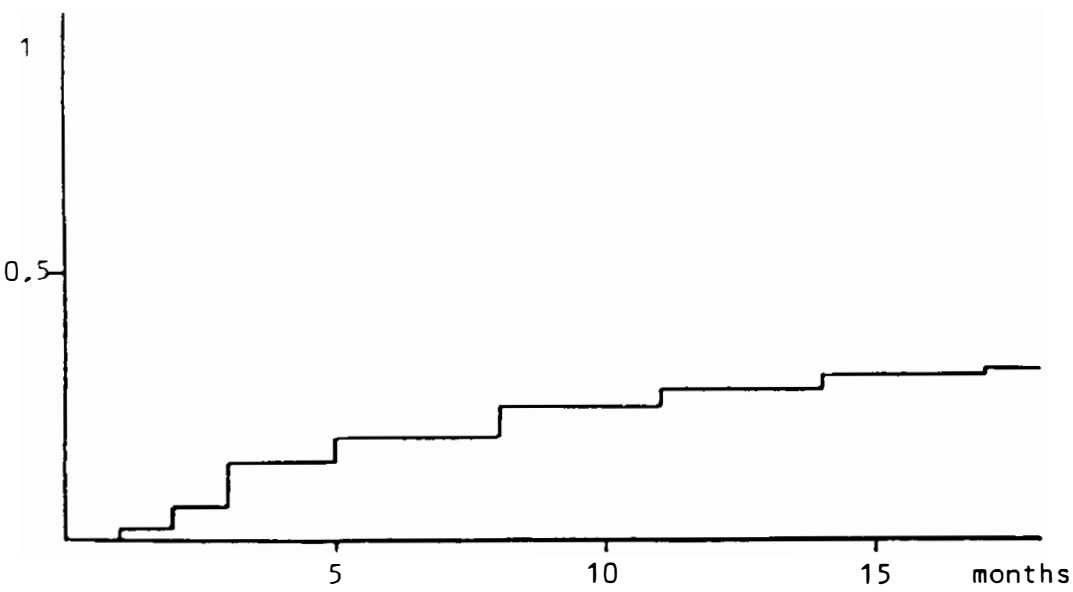

Figure 4 Walking status evolution. 
shows that we have considered all consecutive admissions. The published series that show the prevalence of complete lesions in paraplegics and incomplete lesions in tetraplegics, come from secondary hospitalisation in re-education centres.

The interest of the YSS is to put on a scale the incomplete lesions as shown in Figure 3. However, the YSS does not take into account the problem of the incomplete lesions with sacral sparing.

Walking status recovery

We performed a Kaplan-Meier analysis to obtain an estimate of the probability of walking status recovery as a function of time (Kaplan et al., 1958).

People who died $\left(20^{\circ}\right.$ of the population) are included in the present analysis and were considered as 'patients who would never have walked again'. To include them decreases the selection of the population but, also the relative per cent of good results.

The walking ability recovery as a function of time is shown in Figure 4. Twenty eight per cent of the patients who were admitted to hospital on the day of the injury were functional walkers 1 year later.

\section{Prediction of walking status recovery}

Monofactorial analysis. Each of the three selected factors was correlated with the walking status according to Kaplan-Meier. The Log-Rank test was applied for statistical significance.

The initial YSS appears to be a predictive factor of the walking status (LogRank $=64 ; \mathrm{p}=0.0001$ ).

Twenty four per cent of tetraplegic patients and $38 \%$ of paraplegic patients are functional walkers after 1 year. However, this apparent difference is not significant $(\log -\operatorname{Rank}=2.10 ; \mathrm{p}=0.14)$. In other words, tetraplegia and paraplegia have a similar statistical walking status prognosis.

Age does not appear to be a predictive factor of the walking status (LogRank $=0.36 ; p=0.55)$. This rather surprising result led us to further compare the initial YSS as a function of age (Table). The average lesion in young people is more severe than it is in older people. Their YSS is lower. This point explains why age is not a predictive factor of the walking status and focus on the limitation of a monofactorial analysis.

Multifactorial analysis. The Cox model correlates the walking status with a combination of the three factors (Cox, 1970, 1972). The level of the injury is not significant $(p=0.19)$. However, age becomes significant $(p=0.037)$. This method of adjustment validates through a mathematical calculation, as the youngest people have the most severe injuries (Table).

Table Initial YSS as a function of the age

\begin{tabular}{lcc}
\hline Age & YSS $=0$ & Mean YSS \\
\hline $0-19$ years & $67^{\circ}{ }^{\circ}$ & $1 \cdot 5$ \\
$20-30$ years & $52^{\circ}{ }^{\circ}$ & $1 \cdot 9$ \\
$40-59$ years & $50^{\circ}{ }^{\circ}$ & $1 \cdot 8$ \\
$>$ 60 years & $31^{\circ}{ }_{0}$ & 3 \\
\hline
\end{tabular}




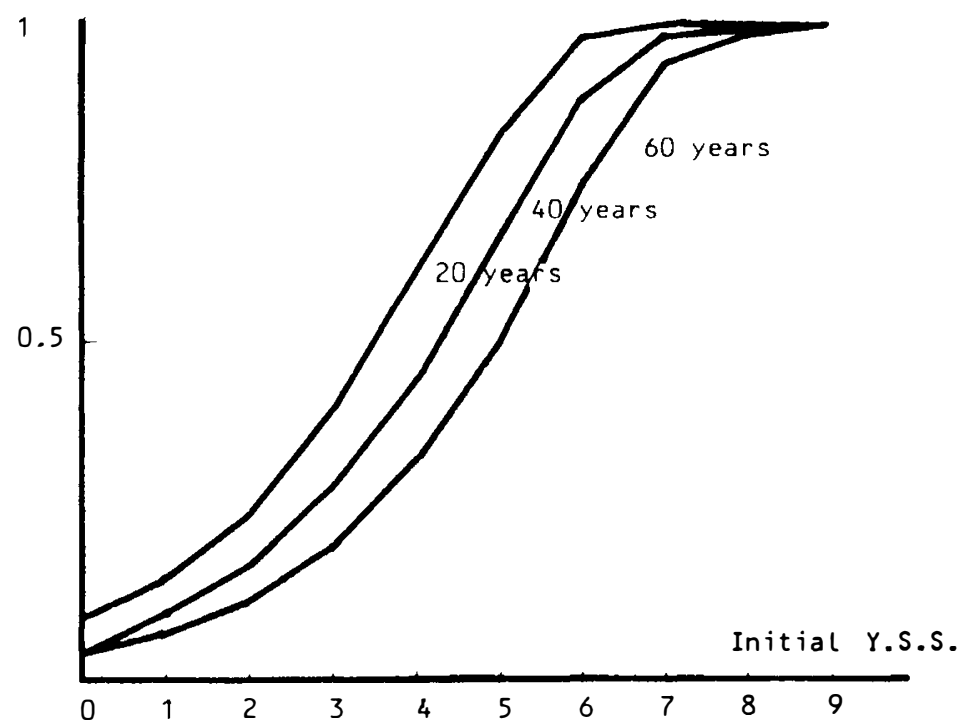

Figure 5 Predicative model of the walking status 1 year after the injury.

The YSS remains very closely correlated with the walking status $(\mathrm{p}=0.0001)$. Thus the YSS is an excellent predictive factor which is the quantitative representation of the initial clinical examination.

Predictive model. Figure 5 is prediction of the walking status 1 year after injury, as a function of the initial YSS for three different ages $(20,40,60$ years).

The three curves are identical. The effect of age is to shift them linearly without altering the slopes. They have a sigmoidal shape; the first part for a YSS $<2$ has a gentle slope, the second part for YSS between 2 and 6 is linear, the third part for YSS $>6$ is flat rapidly reaching $100 \%$.

\section{Discussion}

The study of our population shows that $28^{\circ}{ }_{0}$ of spinal cord injured patients are functional walkers 1 year after injury. This result cannot be compared to those previously published, as, to our knowledge, no other study has studied together paraplegic and tetraplegic patients. Despite the appearance, the statistical analysis allowed it, for we did not find any significant difference between the two populations. Such an affirmation needs to be discussed: the statistical analysis necessarily implies a simplification of clinical information for it studies the prognosis of an 'average person' who is not a given person. The only conclusion we reach, is that statistically the group 'paraplegia' does not recover a better walking status than the group 'tetraplegia', although one can find individual exceptions.

However, our results as a function of the level of the injury may be compared to other reports. Within our population, among 82 cervical lesion, $24 \%$ are functional walkers at 1 year. Frankel et al. (1969), reported that $33 \%$ out of 218 cervical lesions are 'motor useful' after 1 year and 10\% completely recover. 
Maynard et al. (1979), have 103 patients. Thirty nine per cent of the 'sensory incomplete' and $87^{\circ}{ }_{0}$ of the 'motor incomplete' recover a functional walk. These results, which are much better than ours, are explained by the fact that the patients are seen after 1 year without taking into account those who died or were lost during the first year following the injury.

Within our population, among 75 traumatic paraplegics, $38^{\circ}$ o are functional walkers at 1 year. These results are in good agreement with the literature, as the mortality is lower in paraplegia. The population of patients seen after 1 year is not very different from the initial one (Natvig et al., 1979; Van Laere, 1982; Berard et al., 1984; Rodeau and Lecoz, 1986).

To predict the walking status recovery, we only selected three factors (age, level of the lesion, initial YSS). However, multiple injuries, especially head injuries, were also studied. The statistical analysis showed that these were only predictive of survival, but not of walking status (non-published results). Only two factors predicted it: age and the initial YSS. Although age is not the main factor, the YSS is an excellent factor of prognosis as is shown in the curve of the predictive model. The model may be summarised as follows:

- YSS $<2$ : whatever his age, the patient has only a very small chances to walk again and will remain handicapped;

- 2 < YSS 6: the walking status recovery is proportional to the initial YSS; then, age is an essential factor; when YSS is $4.60^{\circ}{ }_{0}$ of the 20 -year-old patients, $45^{\circ}{ }_{0}$ of the 40 -year-old patients and $33^{\circ}{ }_{0}$ of the 60 -year-old patients will walk again. So, the younger the patient is the better the walking status recovery will be;

- YSS > 6: whatever his age, the patient will walk again.

In order to ensure the precision of our predictive model, we made the same statistical analysis to establish another prediction 1 month after the injury. Only 137 patients had survived. The inital YSS remained predictive of the walking status $(\mathrm{p}=0.0001)$. The age was no more significant $(\mathrm{p}=0.51)$, as the oldest patients had died during the first month. This result allowed us to insist on the initial YSS, established by neurological examination. The YSS again appeared to be the best predicting factor of the walking status 1 month after the injury, when spinal shock was absent.

\section{Conclusion}

The initial YSS appears to be an excellent predictive factor of walking status recovery in spinal cord injury. It is reliable and provides precise information which is useful for the team of physicians and surgeons who take care of the patients.

We can appreciate the importance of the initial neurological examination. It is necessary to look for the slightest sign of neurological sparing below the level of the injury. Such information will guide the initial care (especially orthopaedic) and prepare for the functional future of the patient. From an epidemiologic point of view, it points classification of patients according to a scale of prognosis, and thus facilitates the organisation of therapeutic assays. 


\section{References}

Berard E, Depassio J, Pabion C et al. 1984 Influence du traitement initial des lésions vertébrales sur le devenir immédiat et la réadaptation des paraplégies d'origine traumatique (à propos de 100 cas). Acte du congrès AFIGAP, Mulhouse, pp 35-38.

Cherazi B, Wagner F, Collins W et al. 1981 A scale for evaluation of spinal cord injury. fournal of Neurosurgery 54:310-315.

Cox DR 1970 The analysis of binary data. Chapman and Hall, London.

Cox DR 1972 Regression models and life tables. Fournal of the Royal Statistical Society 34:187220.

DARTIGUES JF 1985 Réflexions méthodologiques sur le pronostic, á propos des gliomes cérébraux et des hémorragies cérébrales. Thèse pour le doctorat en biologie humaine. Bordeaux II.

FRANKEL HL, HANCOCK DO, HysLOP G et al. 1969 The value of postural reduction in the initial management of closed injuries of the spine with paraplegia and tetraplegia. Paraplegia 7:179192.

KAPLAN EL, MEIER P 1958 Non parametric estimation from incomplete observations. Fournal of the American Statistical Association 53:457-481.

MAYNARD FM, REYNOLDS GG, FounTAIN S et al. 1979 Neurological prognosis after traumatic quadriplegia. Fournal of Neurosurgery 50:611-616.

Natvig HN, AdAM MR 1979 Ambulation without wheelchairs for paraplegics with complete lesions. Paraplegia 16:142-146.

RODEAU F, LE COX P 1986 La surcharge du handicap chez le paraplégique traumatique. Thèse Bordeaux II.

VAN LAERE M 1982 Emplois des appareils de marche chez le paraplégique. Acte du congrès AFIGAP Bruxelles. 